See discussions, stats, and author profiles for this publication at: https://www.researchgate.net/publication/345017547

\title{
Semiparametric estimation of dynamic discrete choice models
}

Article in Journal of Econometrics · September 2020

DOI: 10.1016/j.jeconom.2020.01.024

CITATIONS

READS

15

3 authors, including:

Matthew Shum

California Institute of Technology

165 PUBLICATIONS 2,903 CITATIONS

SEE PROFILE

Some of the authors of this publication are also working on these related projects:

Project Empirical studies of committee voting View project

Duality in discrete choice models View project 


\title{
SEMIPARAMETRIC ESTIMATION OF DYNAMIC DISCRETE CHOICE MODELS
}

\author{
NICHOLAS BUCHHOLZ ${ }^{\star}$, MATTHEW SHUM $^{\dagger}$, AND HAIQING XU $^{\ddagger}$
}

\begin{abstract}
We consider the estimation of dynamic binary choice models in a semiparametric setting, in which the per-period utility functions are known up to a finite number of parameters, but the distribution of utility shocks is left unspecified. This semiparametric setup differs from most of the existing identification and estimation literature for dynamic discrete choice models. To show identification we derive and exploit a new recursive representation for the unknown quantile function of the utility shocks. Our estimators are straightforward to compute, and resemble classic closed-form estimators from the literature on semiparametric regression and average derivative estimation. Monte Carlo simulations demonstrate that our estimator performs well in small samples.
\end{abstract}

Keywords: Semiparametric estimation, Dynamic discrete choice model, Average derivative estimation, Fredholm integral operators

JEL: C14, D91, C41, L91

Date: Wednesday $29^{\text {th }}$ January, 2020.

*We thank Hassan Afrouzi, Saroj Bhattarai, Stephane Bonhomme, Denis Chetverikov, Kirill Pogorelskiy, Eduardo Souza-Rodrigues, Tang Srisuma, and seminar participants at Academia Sinica, Arizona, Carnegie-Mellon, Chicago, Colorado (Boulder), Duke, Einaudi Institute, Irvine, Washington (Seattle), UT Austin, Wisconsin, Yale, and Texas Metrics Camp for helpful discussions.

^Department of Economics, Princeton University, nbuchholz@princeton.edu.

${ }^{\dagger}$ Division of Humanities and Social Sciences, California Institute of Technology, mshum@caltech.edu.

${ }^{\ddagger}$ Department of Economics, University of Texas at Austin, h.xu@austin.utexas.edu. 


\section{INTRODUCTION}

The dynamic discrete choice (DDC) framework, pioneered by Wolpin (1984), Pakes (1986), Rust $(1987,1994)$, has gradually become the workhorse model for modelling dynamic decision processes in structural econometrics. Such models, which can be considered an extension of McFadden's 1978; 1980 classic random utility model to a dynamic decision setting, have been used to model a variety of economic phenomenon ranging from labor and health economics to industrial organization, public finance, and political economy. More recently, the DDC framework has also been the starting point for the empirical dynamic games literature in industrial organization.

In this paper, we consider identification and estimation of a class of semiparametric dynamic binary choice models in which the utility indices are parametrically specified (as a linear index of observed variables) but the shock distribution is left unspecified. Since the utility shocks are typically interpreted as idiosyncratic and unpredictable shocks to preferences, which cause agents' choices to vary over time even under largely unchanging economic environments, it is reasonable to leave their distribution unspecified. We study conditions under which the model structure (consisting of the finite-dimensional parameters in the utility indices, and the infinitedimensional nonparametric shock distribution) is identified. Our identification argument is constructive, and we propose an estimator based upon it.

The semiparametric DDC framework that we focus on in this paper is novel relative to most of the existing literature on the identification and estimation of DDC models, which considers the case where the utility shocks are fully (parametrically) specified. This reflects an important result in Magnac and Thesmar (2002), who argue that in these models, the single-period utility indices for the choices are (nonparametrically) identifiable only when the distribution of the utility shocks is completely specified. Based on this "impossibility" result, many recent estimators for and applications of DDC models have considered a structure in which the single-period utility indices are left unspecified, but the utility shock distribution is fully specified (and usually logistic, leading to the convenient multinomial logit choice probabilities).

For identification, we derive a new recursive representation for the unknown quantile function of the utility shocks. Accordingly, we obtain a single-index representation for the conditional 
choice probabilities in the model, which permits us to estimate the model using classic estimators from the existing semiparametric binary choice model literature. Specifically, we use Powell, Stock and Stoker's (1989,PSS) kernel-based average-derivative estimator; we show that, under additional mild conditions, our estimator has the same asymptotic properties as PSS's original estimator (which was applied to static discrete-choice models). Moreover, this estimator is computationally quite simple because it can be expressed in closed-form. Monte Carlo simulations demonstrate that our estimator performs well in small samples.

1.1. Literature. This paper builds upon several strands in the existing literature. The semiparametric binary choice literature (e.g. Manski (1975, 1985), Powell, Stock, and Stoker (1989), Ichimura and Lee (1991), Horowitz (1992), and Lewbel (1998), among many others) is an important antecedent. There is a substantive difference, however: because these papers focus on a static model, the shock distribution is treated as a nuisance element. As such, estimation of these shocks is not considered. ${ }^{1}$ In contrast, the shock distribution in a dynamic model must be estimated since it affects the beliefs that decision makers have regarding their future payoffs. Hence, the need to estimate both the utility parameters as well as the shock distribution represents an important point of divergence between our paper and the previous semiparametric discrete choice literature; nevertheless, as we will point out, the estimators we propose take a very similar form to the estimators in these papers.

Srisuma and Linton (2012) pioneered the use of the theory of type 2 integral equations for estimating dynamic discrete-choice models. We show that, besides the Bellman equation, other structural relations in the dynamic model also take the form of type 2 integral equations. In particular, when viewed as a function of the choice probability, the (unknown) quantile function for the utility shocks can also be recursively characterized, which is a key step in our identification and estimation procedure.

There is a growing literature on the identification of dynamic models in which the error distribution is left unspecified. Aguirregabiria (2010) shows the joint nonparametric identification of utilities and the shock distribution in a class of finite-horizon dynamic binary choice models. His identification argument relies on the existence of a final period in the decision problem,

\footnotetext{
${ }^{1}$ An exception is Klein and Spady (1993), who propose a semiparametric maximum likelihood procedure which jointly recovers the distribution of the unobservables and the slope parameters in the single index.
} 
and hence may not apply to infinite-horizon models as considered in this paper. Blevins (2014) considers a very general class of dynamic models in which agents can make both discrete and continuous choices, and the shock distribution can depend on some of the state variables. Under exclusion restrictions, he shows the nonparametric identification of both the per-period utility functions as well as the error distribution. Norets and Tang (2014) focus on the discrete state case, and derive (joint) bounds on the error distribution and per-period utilities which are consistent with an observed vector of choice probabilities. We consider the case with continuous state variables, and discuss nonparametric identification and estimation. ${ }^{2}$ Chen (2017) considers the identification of dynamic models, and, as we do here, obtains estimators for the model parameters which resemble familiar estimators in the semiparametric discrete choice literature. His approach exploits exclusion restrictions (that is, that a subset of the state variables affect only current utility, but not agents' beliefs about future utilities).

What distinguishes our identification approach is that we do not rely on exclusion restrictions, but rather exploit the optimality conditions to derive a new recursive representation of the quantile function for the unobserved shocks in terms of observed quantities. This allows us to identify and estimate both the model parameters as well as the shock distribution and, furthermore, propose a cosed-form estimator for these quantities. ${ }^{3}$

\section{Single Agent Dynamic Binary Choice Model}

Following Rust (1987), we consider a single-agent infinite-horizon binary decision problem. At each time period $t$, the agent observes state variables $X_{t} \in \mathscr{S}_{x} \subseteq \mathbb{R}^{k}$, and chooses a binary decision $Y_{t} \in\{0,1\}$ to maximize her expected utility. The per-period utility is given by

$$
u_{t}\left(Y_{t}, X_{t}, \epsilon_{t}\right)= \begin{cases}W_{1}\left(X_{t}\right)^{\top} \theta_{1}+\epsilon_{1 t}, & \text { if } Y_{t}=1 \\ W_{0}\left(X_{t}\right)^{\top} \theta_{0}+\epsilon_{0 t}, & \text { if } Y_{t}=0\end{cases}
$$

In the above, $W_{0}\left(X_{t}\right) \in \mathbb{R}^{k_{0}}$ (resp. $W_{1}\left(X_{t}\right) \in \mathbb{R}^{k_{1}}$ ) denotes known transformations of the state variables $X_{t}$ which affect the per-period utility from choosing $Y_{t}=0$ (resp. $\left.Y_{t}=1\right)$,

\footnotetext{
${ }^{2}$ With a discrete state space, there can never be point identification when the error distribution has continuous support. When the state space is continuous, however, point identification is possible under some support conditions and a location-scale normalization on the error distribution, as we show.

${ }^{3}$ Miessi Sanches, Silva, and Srisuma (2016) and Dearing (2019) also propose closed-form estimators for DDC models, but their estimators require the researcher to specify the error distribution. Our estimator in contrast, allows the error distribution to be unknown and unspecified by the researcher.
} 
and $\epsilon_{t} \equiv\left(\epsilon_{0 t}, \epsilon_{1 t}\right)^{\top} \in \mathbb{R}^{2}$ are the agent's action-specific payoff shocks, which are observed by the agent but not by the econometrician. The structural parameters which are of interest are $\theta_{d} \in \mathbb{R}^{k_{d}}$, for $d \in\{0,1\}$, along with the distribution of the payoff shocks $F_{\epsilon}$. In what follows, let $W(X) \equiv\left\{W_{0}(X), W_{1}(X)\right\}$ denote the full set of transformed state variables at $X$. For notational simplicity, we will use the shorthand $W_{d}$ for $W_{d}(X)(d=0,1)$ and suppress the explicit dependence upon the state variables $X$ when possible.

This specification of the per-period utility functions in Eq. (1), as single-indices of the transformed state variables $W(X)$ encompasses a majority of the existing applications of dynamic discrete-choice models, and thus imposes little loss in generality. The utility of action 0 is not normalized to be zero for reasons discussed in Norets and Tang (2014). Moreover, let $\beta \in(0,1)$ be the discount factor, which is assumed to be known, ${ }^{4}$ and $f_{X_{t+1}, \epsilon_{t+1} \mid X_{t}, \epsilon_{t}, Y_{t}}$ be the Markov transition probability density function that depends on the state variable as well as the decision.

The agent maximizes the expected discounted sum of the per-period payoffs:

$$
\max _{\left\{y_{t}, y_{t+1}, \ldots\right\}} \mathbb{E}\left\{\sum_{j=0}^{\infty} \beta^{j} u_{t+j}\left(y_{t+j}, X_{t+j}, \epsilon_{t+j}\right) \mid X_{t}, \epsilon_{t}\right\}
$$

We assume stationarity of the problem, which implies that the problem is invariant to the period $t$. Because of this, we can omit the $t$ subscripts and use primes $\left(^{\prime}\right)$ to denote next period values. Let $V(X, \epsilon)$ be the value function given $X$ and $\epsilon$. By Bellman's equation, the value function can be written as

$$
V(X, \epsilon)=\max _{y \in\{0,1\}}\left\{u(y, X, \epsilon)+\beta \mathbb{E}\left[V\left(X^{\prime}, \epsilon^{\prime}\right) \mid X, \epsilon, Y=y\right]\right\}
$$

and then the agent's optimal decision is a stationary Markov process (see e.g. Rust, 1987), given by

$$
Y=\operatorname{argmax}_{y \in\{0,1\}}\left\{u(y, X, \epsilon)+\beta \mathbb{E}\left[V\left(X^{\prime}, \epsilon^{\prime}\right) \mid X, \epsilon, Y=y\right]\right\} .
$$

Unlike much of the existing literature, we do not assume the distribution of the utility shocks $\left(\epsilon_{0 t}, \epsilon_{1 t}\right)$ to be known, but treat their distribution as a nuisance element for the estimation of $\theta$. In a dynamic setting, the distribution of utility shocks also plays the role of agents' beliefs about

\footnotetext{
${ }^{4}$ The assumption that $\beta$ is known is commonplace in the applied DDC literature. See Magnac and Thesmar (2002) and Fang and Wang (2015), among others, for discussion on the identifiability of $\beta$.
} 
the future evolution of state variables (i.e. they are a component in the transition probabilities $f_{X^{\prime}, \epsilon^{\prime} \mid X, \epsilon, Y}$ and hence parametric assumptions on this distribution are not innocuous.

2.1. Characterization of the value function. In this subsection, we characterize the value function $V(X, \epsilon)$ and the expected value function given $X$, i.e., $V^{e}(X) \equiv \mathbb{E}[V(X, \epsilon) \mid X]$. Both value functions are useful to characterize the optimal path in our dynamic model. Let $F_{A}$ and $F_{A \mid B}$ denote the $\mathrm{CDF}$ and the conditional $\mathrm{CDF}$ for generic random variables $A$ and $B$, respectively.

Assumption A (Conditional Independence Assumption). The transition probability satisfies the following condition: $F_{X^{\prime}, \epsilon^{\prime} \mid X, \epsilon, Y}=F_{\epsilon^{\prime}} \times F_{X^{\prime} \mid X, Y}$. Moreover, $F_{\epsilon^{\prime}}=F_{\epsilon}$.

Assumption A is strong, as it establishes that the shocks $\epsilon$ are fully independent of the observed state variables $X^{5}$

Under assumption A, the value function can be written as

$$
V(X, \epsilon)=\max \left\{W_{1}^{\top} \theta_{1}+\epsilon_{1}+\beta \mathbb{E}\left[V\left(X^{\prime}, \epsilon^{\prime}\right) \mid X, Y=1\right], W_{0}^{\top} \theta_{0}+\epsilon_{0}+\beta \mathbb{E}\left[V\left(X^{\prime}, \epsilon^{\prime}\right) \mid X, Y=0\right]\right\} .
$$

Let $\eta=\epsilon_{0}-\epsilon_{1}$. Then the optimal decision maximizing the value function can be written as

$$
Y=\mathbb{1}\left\{\eta \leq \eta^{*}(X)\right\}
$$

where the cutoff $\eta^{*}(X)$ is defined as

$$
\eta^{*}(X) \equiv W_{1}^{\top} \theta_{1}-W_{0}^{\top} \theta_{0}+\beta\left\{\mathbb{E}\left[V\left(X^{\prime}, \epsilon^{\prime}\right) \mid X, Y=1\right]-\mathbb{E}\left[V\left(X^{\prime}, \epsilon^{\prime}\right) \mid X, Y=0\right]\right\}
$$

The starting point for our identification and estimation procedure is a key insight from Srisuma and Linton (2012) which relates an "ex-ante" (or expected) version of the Bellman equation to the mathematical theory of Fredholm integral equations. Let $u^{e}(X)$ be the expected per-period utility conditional on $X$ :

$$
u^{e}(X) \equiv \mathbb{E}\left(\epsilon_{0}\right)+W_{1}^{\top} \theta_{1} \cdot F_{\eta}\left(\eta^{*}(X)\right)+W_{0}^{\top} \theta_{0} \cdot\left[1-F_{\eta}\left(\eta^{*}(X)\right)\right]-\mathbb{E}\left\{\eta \cdot \mathbb{1}\left[\eta \leq \eta^{*}(X)\right]\right\}
$$

where $F_{\eta}$ is the CDF of $\eta$. Thus, the Bellman equation can be rewritten in an "ex-ante" form as

$$
V^{e}(X)=u^{e}(X)+\beta \cdot \mathbb{E}_{\eta^{*}}\left[V^{e}\left(X^{\prime}\right) \mid X\right]
$$

\footnotetext{
${ }^{5}$ This rules out heteroskedasticity in the unobserved shocks, which is accommodated in other papers in the DDC literature (eg. Magnac and Thesmar (2002), Aguirregabiria (2010), Blevins (2014)).
} 
where the notation $\mathbb{E}_{\eta^{*}}$ makes explicit that the expectation is taken over $f_{\eta^{*}}\left(X^{\prime} \mid X\right)$. This is the Markovian transition density for $X$ along the optimal path, which is endogenous as it involves the optimal decision rule characterized by the optimal cutoffs $\eta^{*}$ :

$$
f_{\eta^{*}}\left(X^{\prime} \mid X\right)=\left[1-F_{\eta}\left(\eta^{*}(X)\right)\right] f_{X^{\prime} \mid X, Y}\left(X^{\prime} \mid X, Y=0\right)+F_{\eta}\left(\eta^{*}(X)\right) f_{X^{\prime} \mid X, Y}\left(X^{\prime} \mid X, Y=1\right) .
$$

Eq. (5) is a Fredholm Integral Equation of the second kind (FIE-2); the solution to the integral solution provides an alternative characterization of the value function, as presented in the next Lemma.

Lemma 1. (Srisuma and Linton (2012).) Suppose assumption A holds, and also suppose that, for all $s \geq 1, \mathbb{E}\left(\left\|W_{d}^{[s]}\right\| \mid X\right)<\infty$ a.s., ${ }^{6}$ where the superscript $\left({ }^{[s]}\right)$ denotes the s-period ahead value. Then

$$
V^{e}(x)=u^{e}(x)+\beta \int_{\mathscr{S}_{X}} R^{*}\left(x^{\prime}, x ; \beta\right) \cdot u^{e}\left(x^{\prime}\right) d x^{\prime}, \quad \forall x \in \mathscr{S}_{X},
$$

where $R^{*}\left(x^{\prime}, x ; \beta\right)=\sum_{s=1}^{\infty} \beta^{s-1} f_{X^{[s]} \mid X ; \eta^{*}}\left(x^{\prime} \mid x\right)$ is the resolvent kernel generated by the FIE eq. (5).

Note that we use the superscripted $X^{[s]}$ to denote the $s$-period ahead value of $X$, while the subscripted $X_{s}$ denotes the value of $X$ in period $s$. More succinctly, eq. (6) can be rewritten as

$$
V^{e}(X)=u^{e}(X)+\sum_{s=1}^{\infty} \beta^{s} \cdot \mathbb{E}_{\eta^{*}}\left[u^{e}\left(X^{[s]}\right) \mid X\right] .
$$

In operator notation, eq. (7) denotes exactly the "forward integration" representation of the value function, which is familiar from many two-step procedures for estimating dynamic discrete choice models (see e.g. Hotz and Miller, 1993; Bajari, Benkard, and Levin, 2007; Pakes, Ostrovsky, and Berry, 2007; Hong and Shum, 2010). In the special case when the state variables $X$ are finite and discrete-valued (taking $k<\infty$ values), the Bellman equation is a system of linear equations which can be solved for the value function (cf. Aguirregabiria and Mira, 2007; Pesendorfer and Schmidt-Dengler, 2008) and in that case, the resolvent kernel is just the inverse matrix $\left(I-\beta F_{X^{\prime} \mid X ; \eta^{*}}\right)^{-1}$ where $F_{X^{\prime} \mid X ; \eta^{*}}$ denotes the $k \times k$ transition matrix for $X$ along the optimal path. For notational convenience, in the remainder of this paper, we will omit the subscript of $\eta^{*}$ referring to the optimal dynamic path.

\footnotetext{
${ }^{6}$ This holds, for instance, when $W_{d}(\cdot)$ are bounded functions.
} 
For our semiparametric approach, these developments do not go far enough because they all rely on knowledge of the distribution of utility shocks, $F_{\eta}$. In the following, then, we build on these results to derive another Fredholm integral equation, which characterizes the quantile function of the utility shocks in terms of components which can be estimated directly from the data. This allows us to develop an estimator for dynamic models which do not require knowledge of $F_{\eta}$.

2.2. Optimality Condition. To characterize agents' optimal decisions, the key of our approach is to solve for the cutoff value $\eta^{*}$ that depends on the state variables $X$ (through the transformations $W_{1}(X)$ and $W_{0}(X)$ ). By using eq. (7), along with Lemma 1, eq. (3) becomes

$$
\eta^{*}(X)=W_{1}^{\top} \theta_{1}-W_{0}^{\top} \theta_{0}+\sum_{s=1}^{\infty} \beta^{s}\left\{\mathbb{E}\left[u^{e}\left(X^{[s]}\right) \mid X, Y=1\right]-\mathbb{E}\left[u^{e}\left(X^{[s]}\right) \mid X, Y=0\right]\right\} .
$$

Moreover, let $\phi_{d}(X) \equiv(-1)^{d+1} W_{d}+\sum_{s=1}^{\infty} \beta^{s}\left\{\mathbb{E}\left[W_{d}^{[s]} \mathbb{1}_{Y^{[s]}=d} \mid X, Y=1\right]-\mathbb{E}\left[W_{d}^{[s]} \mathbb{1}_{Y^{[s]}=d} \mid X, Y=\right.\right.$ $0]\}$. Then, it follows from (4),

$$
\begin{aligned}
& \eta^{*}(X)=\phi^{\top}(X) \cdot \theta \\
& \quad-\sum_{s=1}^{\infty} \beta^{s}\left\{\mathbb{E}\left[\eta^{[s]} \mathbb{1}\left(\eta^{[s]} \leq \eta^{*}\left(X^{[s]}\right)\right) \mid X, Y=1\right]-\mathbb{E}\left[\eta^{[s]} \mathbb{1}\left(\eta^{[s]} \leq \eta^{*}\left(X^{[s]}\right)\right) \mid X, Y=0\right]\right\},
\end{aligned}
$$

where $\phi(X)=\left(\phi_{0}^{\top}(X), \phi_{1}^{\top}(X)\right)^{\top}$ and $\theta=\left(\theta_{0}^{\top}, \theta_{1}^{\top}\right)^{\top}$.

Eq. (9) characterizes the optimal decision rule in the single-agent infinite-horizon binary decision problem. Alternatively, we can rewrite it using a resolvent kernel:

$$
\eta^{*}(x)=\phi^{\top}(x) \cdot \theta-\beta \int_{\mathscr{S}_{X}} \mathbb{E}\left[\eta^{\prime} \cdot \mathbb{1}\left(\eta^{\prime} \leq \eta^{*}\left(x^{\prime}\right)\right] \cdot g\left(x^{\prime}, x ; \beta\right) d x^{\prime}, \quad \forall x \in \mathscr{S}_{X},\right.
$$

where $g\left(x^{\prime}, x ; \beta\right)=\sum_{s=1}^{\infty} \beta^{s-1}\left[f_{X[s] \mid X, Y}\left(x^{\prime} \mid x, 1\right)-f_{X[s] \mid X, Y}\left(x^{\prime} \mid x, 0\right)\right]$. Given the structural parameters $\theta_{0}, \theta_{1}, F_{\eta}$ and $f_{X^{\prime} \mid X, Y}$, in principle one can solve the threshold $\eta^{*}(\cdot) .^{7}$

\footnotetext{
${ }^{7}$ However, if one were to use this equation to solve for $\eta^{*}(\cdot)$ via simulation or computation, $g\left(x^{\prime}, x ; \beta\right)$ also contains $\eta^{*}(\cdot)$ implicitly through the transition density $f_{X[s]}{ }_{\mid X, Y}(\cdot \mid \cdot, \cdot)$.
} 


\section{IDENTIFICATION}

Next, we develop an identification strategy that does not involve solving for the optimal decision in the dynamic decision problem. To clarify ideas, we first provide identification of structural parameter $\theta \in \Theta \subseteq \mathbb{R}^{k_{\theta}}$ (where $k_{\theta} \equiv k_{0}+k_{1}$ ) in a fully parametric model, i.e., assuming $F_{\eta}$ is known. Then, we establish semiparametric identification of our model by a two-step approach: we first identify $F_{\eta}$ up to the finite dimensional parameter $\theta$. In the second step, we represent the agent's choice by a single-index representation. Therefore, the identification of $\theta$ follows the literature.

A key feature in our semiparametric identification is that we require (at least) one argument in the state variables $X_{t}$ to have continuous variation, which is also the case in the semiparametric identification of the single-index binary response model in the static setting. See e.g. Manski (1975). Moreover, we show that the quantile function of $F_{\eta}$ is identified on the support of the agent's choice probabilities under a location-scale normalization. This result also corresponds to the findings in static binary response models.

Our identification of $\theta$ is constructive, as we show below that the expectation of $Y$ given $X$, along the optimal dynamic path, is linear in $\theta$. In turn, this leads to an OLS-like (i.e. closed form) estimator for $\theta$. To begin with, we introduce the following assumption.

Assumption B. Let $\eta$ be continuously distributed with the full support $\mathbb{R}$.

Assumption B is a weak condition widely used in semiparametric binary response models (see e.g. Horowitz, 2009). Under assumption $\mathrm{B}, F_{\eta}$ is strictly increasing on its support $\mathbb{R}$. Let $Q$ be the quantile function of $F_{\eta}$, i.e., $Q=F_{\eta}^{-1}$.

Let $p(x)=\mathbb{P}(Y=1 \mid X=x)$, which obtains directly from the data. Under assumption $\mathrm{B}$, $0<p(x)<1$ for all $x \in \mathscr{S}_{X}$ and $\eta^{*}(x)=Q(p(x))$. Moreover, using the substitution $\eta \rightarrow Q(\tau)$, we have

$$
\mathbb{E}\left[\eta \cdot \mathbb{1}(\eta \leq Q(p)]=\int Q(\tau) \mathbb{1}(\tau \leq p) d \tau=\int_{0}^{p} Q(\tau) d \tau\right.
$$

From the above discussion, it is straightforward that we obtain the following lemma. 
Lemma 2. Suppose assumptions $A$ and $B$ hold. Then we have

$$
\begin{array}{r}
Q(p(X))+\sum_{s=1}^{\infty} \beta^{s}\left\{\mathbb{E}\left[\int_{0}^{p\left(X^{[s]}\right)} Q(\tau) d \tau \mid X, Y=1\right]-\mathbb{E}\left[\int_{0}^{p\left(X^{[s]}\right)} Q(\tau) d \tau \mid X, Y=0\right]\right\} \\
=\phi^{\top}(X) \cdot \theta .
\end{array}
$$

Eq. (10) is the key restriction for our identification and estimation analysis, where the number of restrictions equals to the size of the support $\mathscr{S}_{X}$.

When $Q$ is given, then everything in (10) is known except for $\theta$. If, in addition, the matrix $\mathbb{E}\left[\phi(X) \phi^{\top}(X)\right]$ is invertible, then $\theta$ can be estimated using nonlinear least-squares on eq. (10). This approach is related to Pesendorfer and Schmidt-Dengler (2008). ${ }^{8}$

3.1. Semiparametric Identification. Without making any distributional assumptions on $\eta$, we now discuss the identification of $\theta$ as well as $Q$. Intuitively, the number of restrictions imposed by (10) depends on the richness of the support $\mathscr{S}_{X}$. For identification of $Q$ (up to $\theta$ ), we only exploit variation in the choice probabilities $p(X)$.

For notational simplicity, we assume the choice probability $p(X)$ is continuously distributed on a connected interval. ${ }^{9}$

Assumption C. (i) Let $p(X)$ be continuously distributed; (ii) let the support of $p(X)$ be a connected interval, i.e., $[\underline{p}, \bar{p}] \subseteq[0,1]$.

This assumption requires the state variables $X$ to contain some continuous components. Letting $X^{D}$ (resp. $X^{C}$ ) denote the discrete (resp. continuous) components of $X$, a more primitive statement of Assumption $\mathrm{C}$ would be that, for fixed values of the discrete components (say) $X^{D}=x^{d}$, the support of $p\left(X^{C}, x^{d}\right)$ is a closed interval in $[0,1]$. As is well-known, the continuity of covariates is crucial for the semiparametric identification in the static binary response model; this is still the case in our dynamic binary decision model.

\footnotetext{
${ }^{8}$ The full rank of $\mathbb{E}\left[\phi(X) \phi^{\top}(X)\right]$ requires that if the transformed state variables $W_{0}(X)$ and $W_{1}(X)$ contain a common component $W_{c}(X)$, then $\mathbb{E}\left[W_{c}\left(X^{\prime}\right) \mid X, Y=0\right] \neq \mathbb{E}\left[W_{c}\left(X^{\prime}\right) \mid X, Y=1\right]$. This rules out the case that variables without any dynamic transition (e.g. the constant) are included in both transformed state variables. Moreover, we also require that the discount rate $\beta \neq 0$, otherwise $\phi_{0}(X)=W_{0}(X)$ and $\phi_{1}(X)=W_{1}(X)$, which clearly invalidates the rank condition when a common term $W_{c}(X)$ is present.

${ }^{9}$ This interval-support restriction can be relaxed at expositional expense. For instance, suppose $\mathscr{S}_{p(X)}$ is a non-degenerate compact subset of $[0,1]$. All of our identification arguments below still hold by replacing the integral region $[\underline{p}, \bar{p}]$ with $\mathscr{S}_{p(X)}$.
} 
In contrast, when $p(X)$ only has discrete variation (which typically arises when the state variables $X$ themselves have only discrete variation), Norets and Tang (2014) show that the distribution of $\eta$ is partially identified.

For each $p \in[p, \bar{p}]$, let $z(p)=\mathbb{E}[\phi(X) \mid p(X)=p]$. We now take the conditional expectation given $p(X)=p$ on both sides of eq. (10). By the law of iterated expectation, we have

$$
\begin{aligned}
Q(p)+\sum_{s=1}^{\infty} \beta^{s}\left\{\mathbb{E}\left[\int_{0}^{p\left(X^{[s]}\right)} Q(\tau) d \tau \mid p(X)=p, Y=1\right]-\mathbb{E}\left[\int_{0}^{p\left(X^{[s]}\right)} Q(\tau) d \tau \mid p(X)\right.\right. & =p, Y=0]\} \\
& =z(p)^{\top} \cdot \theta .
\end{aligned}
$$

The above discussion is summarized by the following lemma.

Lemma 3. Suppose assumptions $A$ to $C$ hold. Then we have

$$
Q(p)+\beta \int_{\underline{p}}^{\bar{p}} \int_{\underline{p}}^{p^{\prime}} Q(\tau) d \tau \cdot \pi\left(p^{\prime}, p ; \beta\right) d p^{\prime}=z(p)^{\top} \cdot \theta, \quad \forall p \in[\underline{p}, \bar{p}],
$$

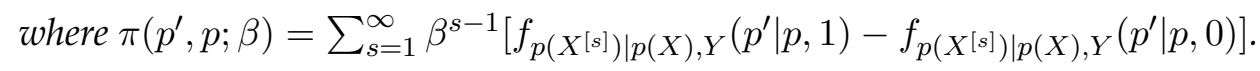

Note that Lemma 3 uses the fact $\int_{\underline{p}}^{\bar{p}} \int_{0}^{p^{\prime}} Q(\tau) d \tau \cdot \pi\left(p^{\prime}, p ; \beta\right) d p^{\prime}=\int_{\underline{p}}^{\bar{p}} \int_{\underline{p}}^{p^{\prime}} Q(\tau) d \tau \cdot \pi\left(p^{\prime}, p ; \beta\right) d p^{\prime}$. By definition, $\pi\left(p^{\prime}, p ; \beta\right)$ is the difference of the discounted aggregate densities of the future choice probabilities, conditional on the current choice probability and (exogenously given) action, which can be obtained directly from the data.

Eq. (11) is also an FIE-2. To see this, let $\Pi\left(p^{\prime}, p ; \beta\right) \equiv \sum_{s=1}^{\infty} \beta^{s-1}\left[F_{p\left(X^{[s]}\right) \mid p(X), Y}\left(p^{\prime} \mid p, 1\right)-\right.$ $\left.\left.F_{p(X[s]}\right) \mid p(X), Y\left(p^{\prime} \mid p, 0\right)\right]$. Then, the second term of eq. (11) can be rewritten as

$$
\begin{aligned}
\int_{\underline{p}}^{\bar{p}} \int_{0}^{p^{\prime}} Q(\tau) d \tau \cdot \pi\left(p^{\prime}, p ; \beta\right) d p^{\prime} & =\int_{0}^{1} Q(\tau) \cdot \int_{\underline{p}}^{\bar{p}} \mathbb{1}\left(\tau \leq p^{\prime}\right) \cdot \pi\left(p^{\prime}, p ; \beta\right) d p^{\prime} d \tau \\
& =-\int_{0}^{1} Q(\tau) \cdot\left[\int_{\underline{p}}^{\bar{p}} \mathbb{1}\left(p^{\prime}<\tau\right) \pi\left(p^{\prime}, p ; \beta\right) d p^{\prime}\right] d \tau \\
& =-\int_{\underline{p}}^{\bar{p}} Q(\tau) \cdot \Pi(\tau, p ; \beta) d \tau
\end{aligned}
$$


where the second step comes from the fact $\int_{\underline{p}}^{\bar{p}} \pi\left(p^{\prime}, p ; \beta\right) d p^{\prime}=0$ and the last step is because $\Pi\left(p^{\prime}, p, \beta\right)=0$ for all $p^{\prime} \notin[\underline{p}, \bar{p}]$. Hence, we obtain the following FIE-2:

$$
Q(p)-\beta \int_{\underline{p}}^{\bar{p}} Q(\tau) \cdot \Pi(\tau, p ; \beta) d \tau=z(p)^{\top} \cdot \theta, \quad \forall p \in[\underline{p}, \bar{p}] .
$$

By solving this equation, we can identify $Q(\cdot)$ on $[\underline{p}, \bar{p}]$ up to the finite dimensional parameter $\theta$.

Assumption D. Let $\beta^{2} \cdot \int_{\underline{p}}^{\bar{p}} \int_{\underline{p}}^{\bar{p}} \Pi^{2}\left(p^{\prime}, p ; \beta\right) d p^{\prime} d p<1$.

Assumption D ensures that the mapping in Eq. (12) is a contraction, so that the solution is unique. This assumption is not a model restriction, but an identification condition, involving both structural primitives as well as variations of observed state variables. Since the discount rate $\beta$ is taken as given in our analysis, this condition is essentially an implicit restriction that $\beta$ be sufficiently far from $1 .{ }^{10}$

Lemma 4. Suppose assumptions $A$ to $D$ hold. Then, $Q$ is point identified on $[\underline{p}, \bar{p}]$ up to the finite dimensional parameter $\theta$ :

$$
Q(p)=\left\{z(p)-\beta \int_{\underline{p}}^{\bar{p}} R\left(p^{\prime}, p ; \beta\right) \cdot z\left(p^{\prime}\right) d p^{\prime}\right\}^{\top} \cdot \theta, \quad \forall p \in[\underline{p}, \bar{p}]
$$

where $R\left(p^{\prime}, p ; \beta\right)=\sum_{s=1}^{\infty}(-\beta)^{s-1} K_{s}\left(p^{\prime}, p ; \beta\right)$, in which $K_{s}\left(p^{\prime}, p ; \beta\right)=\int_{0}^{1} K_{s-1}\left(p^{\prime}, \tilde{p} ; \beta\right) \cdot \Pi(\tilde{p}, p ; \beta) d \tilde{p}$ and $K_{1}\left(p^{\prime}, p ; \beta\right)=\Pi\left(p^{\prime}, p ; \beta\right)$.

The solution (13) is proportional to $\theta$, which is due to the linearity of the FIE system. Therefore, (13) can also be represented by a sequence of "basis" solutions. To see this, let $z_{\ell}(p)$ be the $\ell$-th argument of $z(p)$. For $\ell=1, \cdots, k_{\theta}$, let $b_{\ell}^{*}(\cdot)$ be the (unique) solution to the following equation

$$
b_{\ell}(p)+\beta \int_{\underline{p}}^{\bar{p}} \int_{\underline{p}}^{p^{\prime}} b_{\ell}(\tau) d \tau \cdot \pi\left(p^{\prime}, p ; \beta\right) d p^{\prime}=z_{\ell}(p) .
$$

By a similar argument to Lemma 4, we have

$$
b_{\ell}^{*}(p)=z_{\ell}(p)-\beta \int_{\underline{p}}^{\bar{p}} R\left(p^{\prime}, p ; \beta\right) \cdot z_{\ell}\left(p^{\prime}\right) d p^{\prime}, \quad \forall p \in[\underline{p}, \bar{p}]
$$

\footnotetext{
${ }^{10}$ In our computations, we have never run into convergence problems even for values of $\beta=0.99$.
} 
as the unique solution to $(14)$. Let $\mathcal{B}(\cdot) \equiv\left(b_{1}^{*}(\cdot), \cdots, b_{k_{\theta}}^{*}(\cdot)\right)^{\top}$ be the sequence of solutions supported on $[\underline{p}, \bar{p}]$. Thus, the solution in eq. (13) can be written as

$$
Q(p)=\mathcal{B}(p)^{\top} \cdot \theta, \quad \forall p \in[\underline{p}, \bar{p}] .
$$

By Lemmas 1 to 4, we obtain a single-index representation of the semiparametric dynamic decision model, which is stated in the following theorem.

Theorem 1. Suppose assumptions A to D hold. Then, the agent's dynamic decision can be represented by a static single-index model:

$$
\mathbb{P}(Y=1 \mid X)=F_{\eta}\left(m(X)^{\top} \cdot \theta\right)
$$

where

$$
m(X)=\phi(X)-\sum_{s=1}^{\infty} \beta^{s}\left\{\mathbb{E}\left[\int_{\underline{p}}^{p\left(X^{[s]}\right)} \mathcal{B}(\tau) d \tau \mid X, Y=1\right]-\mathbb{E}\left[\int_{\underline{p}}^{p\left(X^{[s]}\right)} \mathcal{B}(\tau) d \tau \mid X, Y=0\right]\right\},
$$

or alternatively, $m(X)=\mathcal{B}(p(X))$.

Because $\mathbb{P}(Y=1 \mid X)=F_{\eta}(Q(p(X)))$, Theorem 1 obtains by combining eqs. (10) and (15). Given the identification of $\mathcal{B}(\cdot)$ on the support $[\underline{p}, \bar{p}], m(\cdot)$ is then constructively identified on $\mathscr{S}_{X}$. Therefore, the identification of $\theta$ simply follows the single-index model literature, see e.g. Manski $(1975,1985)$.

It is worthing noting that any constant term in $W_{d}$ remains a constant in the transformed linear-index $m(X)$. In other words, suppose, w.l.o.g., $W_{11}=1$. Then the corresponding argument in $m(X)$ also equals 1 . To see this, the first argument in $\phi(X)$ is given by

$$
1+\sum_{s=1}^{\infty} \beta^{s}\left\{\mathbb{E}\left[p\left(X^{[s]}\right) \mid X, Y=1\right]-\mathbb{E}\left[p\left(X^{[s]}\right) \mid X, Y=0\right]\right\},
$$

which thereafter implies

$$
z_{1}(p)=1+\sum_{s=1}^{\infty} \beta^{s}\left\{\mathbb{E}\left[p\left(X^{[s]}\right) \mid p(X)=p, Y=1\right]-\mathbb{E}\left[p\left(X^{[s]}\right) \mid p(X)=p, Y=0\right]\right\} .
$$

Using (14), one can verify that the solution is: $b_{1}^{*}(\cdot)=1$. Then, we plug this solution into the the first element of $m(X)$, which gives us $m_{1}(X)=1$. By a similar argument, a constant in $W_{0}$ implies the corresponding term in $m(X)$ equals -1 . 
By a similar argument as in the static binary response model literature, the index parameter $\theta$ is identified up to location and scale in the semiparametric setting. For notational simplicity, hereafter we assume the state vector $X$ does not include a constant term in the semiparametric setting. ${ }^{11}$ Moreover, we will introduce a scale normalization on $\theta$ which is also standard in the literature.

Assumption E. We denote the first argument of $m(X)$ by $m_{1}(X)$ and the rest by $m_{-1}(X)$. Moreover, let $m_{1}(X)$ be continuously distributed on an interval given $m_{-1}(X)$ which is a vector of either discrete and/or continuous random variables. Let $f_{m_{1}(X) \mid m_{-1}(X)}$ be the conditional $p d f$. Moreover, the matrix $\mathbb{E}\left[m(X) m(X)^{\top}\right]$ is invertible.

In Assumption E, the first half condition requires at least one argument in $X_{1}$ to be continuously distributed conditional on others; this rules out cases where, e.g. all the state variables are functions of a single variable $X_{1}$ (as in (Rust, 1987), where mileage and mileage-squared enter as state variables). The second half of Assumption $\mathrm{E}$ is a testable rank condition. Assumption $\mathrm{E}$ is a strong assumption, but almost indispensable in the semiparametric single index model literature; See Horowitz (2009).

Assumption F. Let $\|\theta\|=1$.

Assumption F is a scale normalization, which has also been used in PSS. We implicitly normalize our location term by 0 , since neither $W_{0}$ nor $W_{1}$ contains a constant term.

Theorem 2. Suppose assumptions $A$ to F hold. Then, the structural parameter $\theta$ is point identified. Furthermore, $F_{\eta}$ is identified by $F_{\eta}(t)=\mathbb{P}\left(Y=1 \mid m(X)^{\top} \theta=t\right)$ for any $t \in \mathscr{S}_{m(X) \mathrm{\top} \theta}$.

Our identification approach for the semiparametric binary dynamic discrete choice model shares many similarities with identification strategies for static semiparametric binary response models, which we briefly discuss here. For identification, we rely explicitly on variation in the choice probabilities $p(X)$. When $X$ is multi-dimensional, then there can exist open sets $\tilde{X} \in \mathcal{X}$ such that the choice probabilities are the same for all $x \in \tilde{X}: p\left(x_{1}\right)=p\left(x_{2}\right)$ for all $x_{1}, x_{2} \in \tilde{X}$. Thus, by the threshold-crossing nature of optimal decisions, agents with $x \in \tilde{X}$ have

\footnotetext{
${ }^{11}$ In our semiparametric setting, any constant term in the utility function will be absorbed by the error term since the distribution of the latter is left unspecified.
} 
the same cutoff $\eta^{*}(x)$ and, under the index assumption on the per-period utilities, we derived that $\eta^{*}(x)=m(x)^{\prime} \theta$, for a vector of functions $m(x)$ which can be estimated directly from the data. Hence, $\theta$ is identified from the equality restrictions $m\left(x_{1}\right)^{\prime} \theta=m\left(x_{2}\right)^{\prime} \theta$ for all $x_{1}, x_{2} \in \tilde{X}$, which do not involve the unknown distribution function of $\eta$. (The normalization $\|\theta\|=1$ eliminates the trivial solution $\theta=0$ to this estimating equation.)

For comparison, Manski's (1988) identification argument for semiparametric static binary response model also relies on a similar argument. Agents with $x \in \tilde{X}$ have the same choice probability $p(x)$ and hence the same cutoff, which in the static case (given the index assumption) is just equal to $x^{\prime} \theta$. Then identification derives from the estimating equation $x_{1}^{\prime} \theta=x_{2}^{\prime} \theta$ for all $x_{1}, x_{2} \in \tilde{X}$.

\section{SEMiparametric Estimation}

In this section, we describe and motivate the semiparametric estimation of our structural model. As is commonly encoutered in the empirical literature on dynamic models, we assume the researcher has panel data on choices and states $\left.\left\{\left(Y_{i t}, X_{i t}^{\top}\right)\right)^{\top}: i=1, \cdots, N ; t=1, \cdots, T\right\}$ where $i$ denotes agents and $t$ denotes periods. For illustration purpose, let $T=2$. Since the underlying dynamic optimization model implies that $\left(Y_{i t}, X_{i t}\right)$ evolve as a stationary (timeinvariant) first-order Markov process, we can identify the conditional choice probabilities $p(Y \mid X)$ and state transitions $f_{X^{\prime} \mid X, Y}$ directly from the data, as detailed below. ${ }^{12}$

Throughout, we use $K$ and $h$ to denote a Parzen-Rosenblatt kernel and a bandwidth, respectively. Moreover, we assume all variables in $X$ are continuously distributed for expositional convenience. ${ }^{13}$ First, we nonparametrically estimate the choice probabilities $p$, (conditional) density functions $f_{X^{\prime} \mid X}$ and $f_{X^{\prime} \mid X, Y}$. This estimates will be used for constructing $m(\cdot)$ later. Let $f_{X}$ be the density function of $X$. Throughout, We assume (i) $f_{X}$ has a convex and compact support, denoted by $\mathscr{S}_{X}$; (ii) $\inf _{x \in \mathscr{S}_{X}} f_{X} \geq \underline{f}$ for some $\underline{f}>0$; (iii) $f_{X}, p$ and $f_{X^{\prime} \mid X, Y}$ are $\iota>2$

\footnotetext{
${ }^{12} \mathrm{~A}$ similar situation arises if the researcher has access to a single long time series with $T \rightarrow \infty$, given minor adjustments to the assumptions.

${ }^{13} \mathrm{~A}$ mixture of continuous and discrete regressors can be accommodated at the expense of notation.
} 
times differentiable on $\mathscr{S}_{X}$ with bounded $\iota$-partial derivatives. Let

$$
\hat{p}(x)=\frac{\sum_{i=1}^{N} Y_{i 1} \times K\left(\frac{X_{i 1}-x}{h_{X}}\right)}{\sum_{i=1}^{N} K\left(\frac{X_{i 1}-x}{h_{X}}\right)},
$$

where we choose an undersmoothing bandwidth, i.e., $h_{X}=1.06 \times \hat{\sigma}_{X} \times N^{-\frac{1}{2 \iota-\iota \epsilon}} ; \hat{\sigma}_{X}$ is the sample standard deviation of $X_{i 1}, \iota_{\epsilon}\left(\iota_{\epsilon}>0\right)$ is an arbitrary small positive number, satisfying $\iota>k+2+0.5 \iota_{\epsilon} \cdot{ }^{14}$ The kernel function $K$ is of order $\iota$. By using a higher order kernel, we ensure the estimation bias of $\hat{p}(\cdot)$ is of order $o\left(N^{-1 / 2}\right)$, and that the variance of the estimator becomes the leading term in its asymptotic performance. The restriction on the choice of bandwidth (i.e. underersmoothing) ensures that the root mean square error in the estimation of $p(\cdot)$ diminishes at rate $\ln N \times N^{-\frac{\iota-0.5 \iota \epsilon-0.5 k}{2 \iota-\iota \epsilon}}$ (faster than $N^{-1 / 4}$ ). See e.g. Hansen (1995) for more detailed results. In addition, the support $[\underline{p}, \bar{p}]$ of $p(X)$ can be estimated by $\left[\min _{i \leq N} \hat{p}\left(X_{i 1}\right), \max _{i \leq N} \hat{p}\left(X_{i 1}\right)\right]$. For notation simplicity, let $\hat{p}_{i 1} \equiv \hat{p}\left(X_{i 1}\right)$.

Moreover, we estimate $f_{X^{\prime} \mid X}$ and $f_{X^{\prime} \mid X, Y}$ respectively by

$$
\begin{aligned}
& \hat{f}_{X^{\prime} \mid X}\left(x^{\prime} \mid x\right)=\frac{\sum_{i=1}^{N} K\left(\frac{X_{i 2}-x^{\prime}}{h_{X}}\right) K\left(\frac{X_{i 1}-x}{h_{X}}\right)}{\sum_{i=1}^{N} K\left(\frac{X_{i 1}-x}{h_{X}}\right)}, \\
& \hat{f}_{X^{\prime} \mid X, Y}\left(x^{\prime} \mid x, d\right)=\frac{\sum_{i=1}^{N} K\left(\frac{X_{i 2}-x^{\prime}}{h_{X d}}\right) K\left(\frac{X_{i 1}-x}{h_{X d}}\right) \mathbb{1}\left(Y_{i 1}=d\right)}{\sum_{i=1}^{N} K\left(\frac{X_{i 1}-x}{h_{X d}}\right) \mathbb{1}\left(Y_{i 1}=d\right)}, d=0,1,
\end{aligned}
$$

where $h_{X d}=1.06 \times \hat{\sigma}_{X d} \times N_{d}^{-\frac{1}{2 \iota-\iota \epsilon}} ; \hat{\sigma}_{X d}$ is the sample standard deviation of $X_{i 1}$ given $Y_{i t}=d$, and $N_{d}=\sum_{i=1}^{N} \mathbb{1}\left(Y_{i 1}=d\right)$. The asymptotic properties of $\hat{f}_{X^{\prime} \mid X}(\cdot \mid \cdot)$ and $\hat{f}_{X^{\prime} \mid X, Y}(\cdot \mid \cdot, d)$ are similar to $\hat{p}$.

Then we estimate the following functionals of $f_{X^{\prime} \mid X}$ and $f_{X^{\prime} \mid X, Y}$ :

$$
\begin{aligned}
\varphi\left(x^{\prime} \mid x\right) & \equiv \sum_{s=1}^{\infty} \beta^{s-1} f_{X^{[s]} \mid X}\left(x^{\prime} \mid x\right), \forall x^{\prime}, x \\
\delta\left(x^{\prime} \mid x\right) & \equiv \sum_{s=1}^{\infty} \beta^{s-1}\left[f_{X^{[s]} \mid X, Y}\left(x^{\prime} \mid x, 1\right)-f_{X^{[s]} \mid X, Y}\left(x^{\prime} \mid x, 0\right)\right], \forall x^{\prime}, x .
\end{aligned}
$$

\footnotetext{
${ }^{14}$ Note that in estimating $\hat{p}(x)$, we do not use observations $\left\{Y_{i 2}: i \leq N\right\}$; this is for convenience, as it is possible to include this additional information to our estimation procedure at the expense of notation and exposition.
} 
By definition, $\varphi$ is the "discounted sum" of $f_{X[s]}$, which leads to an FIE-2 system:

$$
\varphi\left(x^{\prime} \mid x\right)=f_{X^{\prime} \mid X}\left(x^{\prime} \mid x\right)+\beta \int \varphi\left(x^{\prime} \mid \tilde{x}\right) f_{X^{\prime} \mid X}(\tilde{x} \mid x) d \tilde{x}, \forall x^{\prime}, x \in \mathscr{S}_{X} .
$$

This suggests a plug-in estimator: Let $\hat{\varphi}$ solve

$$
\hat{\varphi}\left(x^{\prime} \mid x\right)=\hat{f}_{X^{\prime} \mid X}\left(x^{\prime} \mid x\right)+\beta \int \hat{\varphi}\left(x^{\prime} \mid \tilde{x}\right) \hat{f}_{X^{\prime} \mid X}(\tilde{x} \mid x) d \tilde{x}, \forall x^{\prime}, x .
$$

As standard in the FIE-2 literature, a numerical solution/estimator obtains by using the iteration method: Let $\hat{\varphi}^{[0]}=\hat{f}_{X^{\prime} \mid X}$. Then we set

$$
\hat{\varphi}^{[1]}\left(x^{\prime} \mid x\right)=\hat{f}_{X^{\prime} \mid X}\left(x^{\prime} \mid x\right)+\beta \int \hat{\varphi}^{[0]}\left(x^{\prime} \mid \tilde{x}\right) f_{X^{\prime} \mid X}(\tilde{x} \mid x) d \tilde{x}, \forall x, x^{\prime} .
$$

Repeat such an iteration until it converges, then we obtain $\hat{\varphi}=\hat{\varphi}^{[\infty]}$.

By a similar argument, we have

$\delta\left(x^{\prime} \mid x\right)=f_{X^{\prime} \mid X, Y}\left(x^{\prime} \mid x, 1\right)-f_{X^{\prime} \mid X, Y}\left(x^{\prime} \mid x, 0\right)+\beta \int \varphi\left(x^{\prime} \mid \tilde{x}\right)\left[f_{X^{\prime} \mid X, Y}(\tilde{x} \mid x, 1)-f_{X^{\prime} \mid X f_{X^{\prime} \mid X, Y}, Y}(\tilde{x} \mid x, 0)\right] d \tilde{x}$, which is estimated by $\hat{\delta}\left(x^{\prime} \mid x\right)=\hat{f}_{X^{\prime} \mid X, Y}\left(x^{\prime} \mid x, 1\right)-\hat{f}_{X^{\prime} \mid X, Y}\left(x^{\prime} \mid x, 0\right)+\beta \int \hat{\varphi}\left(x^{\prime} \mid \tilde{x}\right)\left[\hat{f}_{X^{\prime} \mid X, Y}(\tilde{x} \mid x, 1)-\hat{f}_{X^{\prime} \mid X, Y}(\tilde{x} \mid x, 0)\right] d \tilde{x}$.

Note that both $\hat{\varphi}$ and $\hat{\delta}$ are functionals of $\hat{f}_{X^{\prime} \mid X}$ and $\hat{f}_{X^{\prime} \mid X, Y}$, Hadamard directionally differentiable. By the functional delta method, the asymptotic behaviors of all these second stage estimators can be derived from $\hat{f}_{X^{\prime} \mid X}-f_{X^{\prime} \mid X}$ and $\hat{f}_{X^{\prime} \mid X, Y}-f_{X^{\prime} \mid X, Y}$.

Next, we estimate $\phi_{d}(\cdot)$ and $m_{\ell}(\cdot)$. Note that

$$
\phi_{d}(x)=(-1)^{d+1} W_{d}(x)+\beta \int \mathbb{E}\left(W_{d} \mathbb{1}_{Y=d} \mid X=x^{\prime}\right) \times \delta\left(x^{\prime} \mid x\right) d x^{\prime},
$$

in which $\mathbb{E}\left(W_{d} \mathbb{1}_{Y=d} \mid X=x^{\prime}\right)=W_{d}\left(x^{\prime}\right) p^{d}\left(x^{\prime}\right)\left[1-p\left(x^{\prime}\right)\right]^{1-d}$. Therefore, let

$$
\hat{\phi}_{d}(x)=(-1)^{d+1} W_{d}(x)+\beta \int W_{d}\left(x^{\prime}\right) \hat{p}^{d}\left(x^{\prime}\right)\left[1-\hat{p}\left(x^{\prime}\right)\right]^{1-d} \times \hat{\delta}\left(x^{\prime}, \mid x\right) d x^{\prime}
$$


Again, by the functional delta method, the asymptotic behavior of $\hat{\phi}_{d}-\phi_{d}$ depends on $\hat{p}-p$, $\hat{f}_{X^{\prime} \mid X, Y}-\hat{f}_{X^{\prime} \mid X, Y}$ and $\hat{\varphi}-\varphi$. To estimate $b_{\ell}^{*}$, note that (12) implies

$$
b_{\ell}^{*}(p)-\beta \int_{\underline{p}}^{\bar{p}} b_{\ell}^{*}(\tau) \times \Pi(\tau, p ; \beta) d \tau=z_{\ell}(p), \forall p \in[\underline{p}, \bar{p}],
$$

in which $\Pi$ and $z_{\ell}$ are estimated by

$$
\begin{aligned}
& \hat{\Pi}(\tau, p ; \beta)=\frac{\sum_{i=1}^{N} \int \mathbb{1}\left[\hat{p}\left(x^{\prime}\right)<\tau\right] \hat{\delta}\left(x^{\prime} \mid X_{i 1}\right) d x^{\prime} \times K_{p}\left(\frac{\hat{p}\left(X_{i 1}\right)-p}{h_{p}}\right)}{\sum_{i=1}^{N} K_{p}\left(\frac{\hat{p}\left(X_{i 1}\right)-p}{h_{p}}\right)} ; \\
& \hat{z}_{\ell}(p)=\frac{\sum_{i=1}^{N} \hat{\phi}_{\ell}\left(X_{i 1}\right) K_{p}\left(\frac{\hat{p}\left(X_{i 1}\right)-p}{h_{p}}\right)}{\sum_{i=1}^{N} K_{p}\left(\frac{\hat{p}\left(X_{i 1}\right)-p}{h_{p}}\right)} .
\end{aligned}
$$

where $h_{p}=1.06 \times \hat{\sigma}_{p} \times N^{-\frac{1}{2 \iota-\iota_{\epsilon}}}$ is an underersmoothing bandwidth, $\hat{\sigma}_{p}$ is the sample standard deviation of $p\left(X_{i 1}\right)$, and the order of the kernel function $K_{p}$ is $\iota$. We can apply e.g. Kristensen (2010), Mammen, Rothe, Schienle, et al. (2012) and Mammen, Rothe, and Schienle (2016) to derive the asymptotic performance of the above estimators. ${ }^{15}$

Moreover, we estimate $b_{\ell}^{*}(\cdot)$ by solving

$$
\hat{b}_{\ell}^{*}(p)-\beta \int_{\underline{p}}^{\bar{p}} \hat{b}_{\ell}^{*}(\tau) \times \hat{\Pi}(\tau, p ; \beta) d \tau=\hat{z}_{\ell}(p), \forall p \in\left[\underline{p}+h_{p}, \bar{p}-h_{p}\right] .
$$

Again, we apply an iteration algorithm to this FIE-2 system.

We are now ready to construct the single-index variables $m\left(X_{i 1}\right)$ : For $\ell=1, \cdots, k_{\theta}$, let

$$
\hat{m}_{\ell}\left(X_{i 1}\right)=\hat{b}_{\ell,-i}^{*}\left(\hat{p}_{-i}\left(X_{i 1}\right)\right), \quad i=1, \cdots, N
$$

where $\hat{b}_{\ell,-i}^{*}$ and $\hat{p}_{-i}$ are the so-called leave-one-out estimators, defined the same as $\hat{b}_{\ell}^{*}$ and $\hat{p}$ except for excluding observation $i$ for their expressions.

Finally, we apply PSS to estimate $\theta$ (up to scale), which takes a closed-form expression and does not involve a trimming mechanism. ${ }^{16}$ Specifically, we define

$$
\hat{\theta}=-\frac{2}{N(N-1)} \sum_{i=1}^{N} \sum_{j \neq i}\left[\frac{1}{h_{\theta}^{k_{\theta}+1}} \times \nabla K_{\theta}\left(\frac{\hat{m}\left(X_{i 1}\right)-\hat{m}\left(X_{j 1}\right)}{h_{\theta}}\right) \times Y_{j 1}\right] .
$$

\footnotetext{
${ }^{15}$ In particular, Mammen, Rothe, Schienle, et al. (2012) uses empirical process theory to provide a stochastic expansion that characterizes the influence of the generator regressor in the kernel function for asymptotic analysis.

${ }^{16}$ One could also use alternative methods e.g. Klein and Spady (1993) and Ichimura (1993) to estimate $\theta$.
} 
Following the standard kernel regression literature, we can show $\hat{\theta}$ is consistent given that $\sup _{x \in \mathscr{S}_{X}}|\hat{m}(x)-m(x)|=o_{p}\left(h_{\theta}\right), h_{\theta} \rightarrow 0$ and $N h_{\theta}^{k_{\theta}+1} \rightarrow \infty$ as $N \rightarrow \infty$.

With these considerations in place, we next establish $\sqrt{N}$-consistency of $\hat{\theta}$. Following PSS, we need to choose a higher-order kernel $K_{\theta}$ and an undersmoothing bandwidth $h_{\theta}$. However, it is more delicate in our setting because of the generated regressor $\hat{m}\left(X_{1}\right)$ contained in the kernel function of our estimator (16). Due to the first-stage estimation error, we must make the following additional assumptions on the convergence of $\hat{m}\left(X_{1}\right)$ to $m\left(X_{1}\right)$ :

Assumption G. (i) $\mathbb{E}\|\hat{m}(X)-m(X)\|^{2}=o\left(N^{-\frac{1}{2}} h_{\theta}^{3}\right)$;

(ii) $\mathbb{E}\|\mathbb{E}[\hat{m}(X) \mid X]-m(X)\|=o\left(N^{-\frac{1}{2}} h_{\theta}^{2}\right)$.

Assumption G encompasses high-level conditions that could be further established under primitive conditions. In particular, Assumption $\mathrm{G}(\mathrm{i})$ requires $\hat{m}(\cdot)$ to converge to $m(\cdot)$ faster than $N^{-\frac{1}{4}}$. By Assumption G(ii), the bias term in the estimation of $m$ uniformly converges to zero faster than $N^{-\frac{1}{2}}$. Hence, we need to use a higher order kernel in the estimation of $m(\cdot)$. Assumption $\mathrm{G}$ is standard in the literature for the regular convergence of finite-dimensional parameters in semiparametric models (e.g. Ai and Chen, 2003), except for the polynomial terms of $h_{\theta}$ in the $o(\cdot)$ or $o_{p}(\cdot)$ which arises due to the average derivate estimator in the second stage. Let $f_{m}$ be the density function of $m(X)$.

Assumption H. (i) The support of $f_{m}$ is convex subset of $\mathbb{R}^{d_{\theta}}$ with nonempty interior; (ii) The density function $f_{m}$ is continuous in the components, so that $f_{m}(m)=0$ for all $m \in \mathbb{R}^{d_{\theta}}$ on the boundary of its support; (iii) $f_{m}(\cdot)$ and $\mathbb{P}(Y=1 \mid m(X)=\cdot$ ) are continuously differentiable on its support. (iv) The components of the random vector $\frac{\partial \mathbb{P}(Y \mid m(X))}{\partial m}$ and random matrix $\frac{\partial f_{m}}{\partial m}$ have finite second moments. In addition, $\frac{\partial f_{m}}{\partial m}$ and $\frac{\partial \mathbb{P}(Y \mid m(X)) f_{m}}{\partial m}$ satisfy the Lipschitz conditions in PSS; (v) Let $k^{*}=\frac{k_{\theta}+3+\mathbb{1}\left(k_{\theta} \text { is even }\right)}{2}$. All partial derivatives of $f_{m}$ of order $k^{*}+1$ exist; and the expectation $\mathbb{E}\left(Y \frac{\partial^{k} f_{m}}{\partial m_{\ell_{1}} \cdots \partial m_{\ell_{k}}}\right)$ exists for all $k \leq k^{*}+1$; (vi) Let $\eta$ be continuously distributed with density function $f_{\eta}$.

Assumption I. $h_{\theta}=N^{-\frac{1}{\gamma}}$ where $k_{\theta}+2<\gamma<k_{\theta}+3+\mathbb{1}\left(k_{\theta}\right.$ is even $)$.

Assumption J. The support of the kernel function $K_{\theta}$ is a convex subset of $\mathbb{R}^{k_{\theta}}$ with nonempty interior, with the origin as an interior point; $K_{\theta}$ is a bounded differentiable function that obeys: $\int K_{\theta}(u) d u=1$, 
$K_{\theta}(u)=0$ for all $u$ belongs to the boundary of its support, $K_{\theta}(u)=K_{\theta}(-u)$ and

$$
\begin{aligned}
& \int u_{1}^{\ell_{1}} \cdots u_{k_{\theta}}^{\ell_{\rho^{\prime}}} K_{\theta}(u) d u=0, \text { for } \ell_{1}+\cdots+\ell_{\rho^{\prime}}<\frac{k_{\theta}+3+\mathbb{1}\left(k_{\theta} \text { is even }\right)}{2}, \text { and } \\
& \int u_{1}^{\ell_{1}} \cdots u_{k_{\theta}}^{\ell_{\rho^{\prime}}} K_{\theta}(u) d u \neq 0, \text { for } \ell_{1}+\cdots+\ell_{\rho^{\prime}}=\frac{k_{\theta}+3+\mathbb{1}\left(k_{\theta} \text { is even }\right)}{2} .
\end{aligned}
$$

where $u_{\ell}$ is the $\ell-$ th argument of $u . K_{\theta}$ is three times differentiable and $\int\|u\| \times\left\|\nabla^{3} K(u)\right\| d u<\infty$. Moreover, all moments of $K_{\theta}$ of order $k^{*}$ exist.

Assumptions $\mathrm{H}$ to J are regularity conditions introduced by PSS. Specifically, Assumption H represents the regularity conditions, e.g., on the smoothness of the underlying structural components of our model. Assumptions I and J are made for the choice of bandwidth and kernel, respectively, to control the bias term in the estimation of $\theta$ and the issue due to the generated regressor $\hat{m}$. The restriction on the bandwidth Assumption I implies that $h_{\theta}$ is not an optimal bandwidth sequence (rather it is undersmoothed) such that the bias of estimating $\theta$ goes to zero faster than $\sqrt{N}$.

Given these assumptions, we can show the following result (the proof is in the appendix):

Theorem 3. Suppose assumptions G to J hold. Then, for some scalar $\lambda>0$ specified below, $\sqrt{N}(\hat{\theta}-\lambda \cdot \theta)$ has a limiting multivariate normal distribution defined in Powell, Stock, and Stoker (1989, Theorem 3.1):

$$
\sqrt{N}(\hat{\theta}-\lambda \cdot \theta) \stackrel{d}{\rightarrow} N(0, \Sigma)
$$

where $\Sigma \equiv 4 \mathbb{E}\left(\zeta \zeta^{\top}\right)-4 \lambda^{2} \theta \theta^{\top}, \zeta=f_{m}\left(m\left(X_{1}\right)\right) f_{\eta}\left(\eta^{*}\left(X_{1}\right)\right) \theta-\left[Y_{1}-F_{\eta}\left(\eta^{*}\left(X_{1}\right)\right)\right] f_{m}^{\prime}\left(m\left(X_{1}\right)\right)$ and $\lambda=\mathbb{E}\left[f_{m}\left(m\left(X_{1}\right)\right) f_{\eta}\left(m\left(X_{1}\right)^{\top} \theta\right)\right]$.

In the above theorem, recall $\mathbb{P}\left(Y_{1}=1 \mid X_{1}\right)=F_{\eta}\left(\eta^{*}\left(X_{1}\right)\right)$ and $\eta^{*}\left(X_{1}\right)=m\left(X_{1}\right)^{\top} \theta$ by Theorem 1 . Our estimator $\hat{\theta}$ (as defined in Eq. (16)) has not imposed the scale restriction in Assumption F; thus $\lambda \in \mathbb{R}$ in the above theorem denotes the probability limit of $\|\hat{\theta}\|$; i.e., $\|\hat{\theta}\|=\lambda+O_{p}\left(N^{-\frac{1}{2}}\right)$. Therefore, by rescaling our estimator $\hat{\theta}$ as $\hat{\theta}^{*}=\hat{\theta} /\|\hat{\theta}\|$, we obtain that

$$
\sqrt{N}\left(\hat{\theta}^{*}-\theta\right) \stackrel{d}{\rightarrow} N\left(0, \Sigma / \lambda^{2}\right)
$$

Given the asymptotic normality established in this section, bootstrap inference is valid for empirical applications. 
Given $\hat{\theta}^{*}$, a nonparametric estimator of $Q(\cdot)$ directly follows from Eq. (13). Namely, let

$$
\hat{Q}(p)=\hat{z}^{\top}(p) \times \hat{\theta}^{*}, \forall p \in\left[\underline{p}+h_{p}, \bar{p}-h_{p}\right] .
$$

Because of the $\sqrt{N}$-consistency of $\hat{\theta}^{*}$, the estimator $\hat{Q}(p)$ is asymptotically equivalent to $\hat{z}^{\top}(p) \times \theta$, which converges at a nonparametric rate. ${ }^{17}$

4.1. Monte Carlo. The focus of our Monte Carlo is on the semiparametric estimation. In our experiments, let $u_{t}\left(0, X_{t}, \epsilon_{t}\right)=\theta_{0}+\epsilon_{0 t}$ and $u_{t}\left(1, X_{t}, \epsilon_{t}\right)=X_{1 t} \theta_{1}+X_{2 t} \theta_{2}+\epsilon_{1 t}$, where $X_{1 t}, X_{2 t}$ are random variables and $\theta_{0}, \theta_{1}, \theta_{2} \in \mathbb{R}$. Moreover, we set the conditional distribution of $X_{t+1}$ given $X_{t}$ and $Y_{t}$ as follows: for $k=1,2$

$$
X_{k, t+1}=\left\{\begin{array}{cl}
X_{k t}+\nu_{k t}, & \text { if } Y_{t}=0 \\
\nu_{k t} & \text { if } Y_{t}=1
\end{array},\right.
$$

where $\nu_{1 t}$ and $\nu_{2 t}$ conform to a Lognormal $(0,1)$ and a Lognormal $(0,4)$ respectively, and $\nu_{1 t} \perp \nu_{2 t}$. Our assumption on the distribution of $\epsilon_{d t}$ will vary across the specifications, as detailed below. We set $\beta=0.9$ and set the parameter values as follows: $\theta_{0}=6, \theta_{1}=0.5$ and $\theta_{2}=0.5$.

Because we cannot estimate the constant $\theta_{0}$ in the semiparametric framework, we treat $\theta_{0}$ as a nuisance parameter. Let $\theta=\left(\theta_{1}, \theta_{2}\right)^{\top}$. $\theta$ is only identified up to scale in the semiparametric setting. To compare the performance of the semiparametric estimators, we assume the scale of $\theta$ is known, i.e., $\|\theta\|=\sqrt{0.5}$, rather than imposing a different normalization, as assumption $\mathrm{F}$.

Based on this setup, Table 1 shows a set of Monte Carlo estimates which examines the performance of our estimator, denoted as BSX, under three specifications. In Specification 1 unobservables are distributed as Type I extreme value, with zero mean and unit variance. This corresponds to the typical assumption made in Rust (1987) and in many other applications of dynamic discrete-choice models. Specification 2 uses data generated from a model in which unobservables are drawn from an equally-weighted two-component mixture of T1EV distributions, with the first (second) component having a mean of $4(-4)$ and standard deviation of 2 (3). Specification 3 is similar, but with mean and variance of $(4,2)$ and $(-3,2)$ across the two components. In these last two specifications, the error distribution is bimodal, and parametric

\footnotetext{
${ }^{17}$ The asymptotic properties of $\hat{z}^{\top}(p)$ can be established by following Guerre, Perrigne, and Vuong (2000), who use nonparametrically estimated pseudo private values to construct a kernel estimator for the density function of bidders' private values in an independent private value auction model.
} 
TABLE 1. Monte Carlo Results

\begin{tabular}{cccccccc}
\hline & \multicolumn{2}{c}{$\begin{array}{c}\text { Specification 1: } \\
\text { Logit }\end{array}$} & \multicolumn{2}{c}{$\begin{array}{c}\text { Specification 2: } \\
\text { Bimodal mixture }\end{array}$} & \multicolumn{2}{c}{$\begin{array}{c}\text { Specification 3: } \\
\text { Bimodal mixture }\end{array}$} \\
Sample size & $\theta_{1}$ & $\theta_{2}$ & $\theta_{1}$ & $\theta_{2}$ & $\theta_{1}$ & $\theta_{2}$ \\
\hline True Value: & 0.5 & 0.5 & 0.5 & 0.5 & 0.5 & 0.5 \\
& & & & & & \\
$N=1000$ & 0.487 & 0.493 & 0.478 & 0.460 & 0.492 & 0.462 \\
& $(0.098)$ & $(0.102)$ & $(0.164)$ & $(0.182)$ & $(0.144)$ & $(0.155)$ \\
& & & & & & \\
$N=2000$ & 0.490 & 0.498 & 0.488 & 0.491 & 0.487 & 0.492 \\
& $(0.078)$ & $(0.058)$ & $(0.098)$ & $(0.076)$ & $(0.092)$ & $(0.074)$ \\
& & & & & & \\
$N=4000$ & 0.495 & 0.498 & 0.498 & 0.491 & 0.497 & 0.492 \\
& $(0.058)$ & $(0.058)$ & $(0.073)$ & $(0.076)$ & $(0.073)$ & $(0.074)$ \\
& & & & & & \\
\hline Logit-MLE & 0.502 & 0.502 & 0.215 & 0.193 & 0.226 & 0.188 \\
$N=4000$ & $(0.031)$ & $(0.029)$ & $(0.02)$ & $(0.013)$ & $(0.018)$ & $(0.01)$ \\
\hline
\end{tabular}

This table presents Monte Carlo results for our BSX estimator across different specifications and sample sizes. Specification 1 uses data generated where unobservables are distributed as Type-I extreme value with mean 0 and variance 1 . Specifications 2 and 3 use data generated from an equally-weighted mixture of two different T1EV distributions. Each row offers estimates for different sample sizes, except the last row which implements a nested fixed-point MLE estimator under a unimodal logit assumption on unobservables. For each sample size, reported estimates and standard deviations (in parentheses) are computed as the mean across 150 simulation draws.

estimation using either the full-solution or CCP-based methods would be challenging, as the model choice probabilities no longer have a closed-form.

Table 1 highlights that our estimator is able to identify and estimate $\theta$ (up to scale) regardless of the underlying distribution of unobservables. Even at smaller sample sizes $(N=1000)$, the estimator performs well, with no marked deterioration relative to larger sample sizes. In the last row, we contrast our results with an MLE estimator under the assumption that unobservables are T1EV (corresponding to Rust's (1987) nested fixed-point estimator). When the model is correctly specified as in Specification 1, the MLE performs well. However when we generate data using the non-logit mixture distributions, as in Specifications 2 and 3, the MLE is misspecified, and we see that this misspecification introduces bias. In contrast to our BSX estimator, which continues 
to recover parameters up to scale, the MLE performs poorly, attributing parameter values which are in neither absolute nor relative terms close to the true values. ${ }^{18}$

Another important benefit of our approach is the short computational time: a single estimation procedure takes about 0.9 seconds when $N=1000$, and grows to about 13 seconds as the sample size increases to $N=4000$. By contrast the MLE estimation of takes close to 30 minutes, as the choice probabilities must be simulated for each trial value of the parameters.

\section{CONCLUSiOnS}

In this paper we consider the estimation of dynamic binary discrete choice models in a semiparametric setting, in which the per-period utility functions are parameterized as singleindex functions, but the distribution of the utility shocks is left unspecified and treated as nuisance components of the model. This setup differs from most of the existing work on estimation and identification of dynamic discrete choice models. For identification, we derive a new recursive representation for the unknown quantile function of the utility shocks; our argument requires no additional exclusion restrictions beyond the conditional independence conditions assumed in the typical parametric dynamic-discrete choice literature (e.g. Rust $(1987,1994))$. Accordingly, we obtain a single-index representation for the conditional choice probabilities in the model, which permits us to estimate the model using classic estimators from the existing semiparametric binary choice literature.

In particular, we use Powell, Stock and Stoker's (1989) kernel-based estimator to estimate the dynamic discrete choice model. We show that the estimator has the same asymptotic properties as PSS's original estimator (for static discrete-choice models), under mild conditions. Significantly, the computational procedure is quite simple, because the estimator for the parameters can be expressed in closed-form. Monte Carlo simulations show that the estimator works well even in moderately-sized samples.

In this paper, we focus on the dynamic binary choice model. An extension to multinomial $(\geq 3)$ choice appears challenging, because our procedure relies strongly on a threshold-crossing property of the optimal decision rule (eqs. (2)-(3)). This is natural for binary choice, but not obviously generalizable to multinomial choice settings.

\footnotetext{
${ }^{18}$ Note that the MLE procedures estimate the constant parameter $\theta_{0}$, which we did not report in Table 1.
} 
In current work, we are using our procedure to estimate a dynamic labor supply model for taxicab drivers, using a large sample of shifts of New York City taxicabs (Buchholz, Shum, and $\mathrm{Xu}$ (2018)). Since our estimator works for any dynamic binary choice problem, it may be fruitfully applied to dynamic entry games which have been estimated in the empirical IO literature (eg. Ryan (2012), Collard-Wexler (2013)), as well as models of technology or new product adoption (eg. Nair (2007), Ryan and Tucker (2012)). We will explore these possibilities in future work.

\section{REFERENCES}

AguirRegabiriA, V. (2010): “Another Look at the Identification of Dynamic Discrete Decision Processes: An Application to Retirement Behavior," Journal of Business and Economic Statistics, 28(2), 1795-1843.

Aguirregabiria, V., AND P. Mira (2007): “Sequential estimation of dynamic discrete games," Econometrica, pp. 1-53.

AI, C., AND X. CHEN (2003): “Efficient estimation of models with conditional moment restrictions containing unknown functions," Econometrica, 71(6), 1795-1843.

BAJARI, P., L. BENKARD, AND J. LeVIN (2007): “Estimating Dynamic Models of Imperfect Competition," Econometrica, 75(5), 1331-1370.

BLEVINS, J. (2014): "Nonparametric identification of dynamic decision processes with discrete and continuous choices," Quantitative Economics, 5, 531-554.

BuchHolz, N., M. SHUM, AND H. XU (2018): “Dymamic Labor Supply of Taxicab Drivers: a Semiparametric Optimal Stopping Model," Working Paper.

CHEN, L.-Y. (2017): “Identification of Discrete Choice Dynamic Programming Models with Nonparametric Distribution of Unobservables," Econometric Theory, 33, 551-577.

COLlARD-WeXLeR, A. (2013): “Demand fluctuations in the ready-mix concrete industry," Econometrica, 81(3), 1003-1037.

DeAring, A. (2019): “Pseudo-Value Functions and Closed-Form CCP Esitmation of Dynamic Discrete Choice Models," Working Paper.

FANG, H., AND Y. WANG (2015): “Estimating Dynamic Discrete Choice Models with Hyperbolic Discounting, with an Application to Mammography Decisions," International Economic Review, 56(2), 565-596.

Guerre, E., I. Perrigne, ANd Q. VuONG (2000): “Optimal Nonparametric Estimation of First-Price Auctions," Econometrica, 68, 525-74. 
HANSEN, B. E. (1995): “Rethinking the univariate approach to unit root testing: Using covariates to increase power," Econometric Theory, 11(5), 1148-1171.

HONG, H., AND M. SHUM (2010): "Pairwise-difference estimation of a dynamic optimization model," Review of Economic Studies, 77(1), 273-304.

HorowitZ, J. L. (1992): “A smoothed maximum score estimator for the binary response model," Econometrica, pp. 505-531.

(2009): Semiparametric and nonparametric methods in econometrics. Springer.

Hotz, V. J., AND R. A. Miller (1993): “Conditional choice probabilities and the estimation of dynamic models," Review of Economic Studies, 60(3), 497-529.

ICHIMURA, H. (1993): "Semiparametric least squares (SLS) and weighted SLS estimation of single-index models," Journal of Econometrics, 58(1), 71-120.

ICHIMURA, H., AND L.-F. LEE (1991): “Semiparametric least squares estimation of multiple index models: single equation estimation," in Nonparametric and semiparametric methods in econometrics and statistics: Proceedings of the Fifth International Symposium in Economic Theory and Econometrics. Cambridge, pp. 3-49.

KLEIN, R., AND R. SPADY (1993): “An efficient semiparametric estimator for binary response models," Econometrica, 61(2), 387-421.

KRISTENSEN, D. (2010): "Pseudo-maximum likelihood estimation in two classes of semiparametric diffusion models," Journal of Econometrics, 156(2), 239-259.

LEWBEL, A. (1998): "Semiparametric latent variable model estimation with endogenous or mismeasured regressors," Econometrica, pp. 105-121.

MAGNAC, T., AND D. Thesmar (2002): “Identifying dynamic discrete decision processes," Econometrica, 70(2), 801-816.

Mammen, E., C. Rothe, And M. Schienle (2016): "Semiparametric estimation with generated covariates," Econometric Theory, 32(5), 1140-1177.

Mammen, E., C. Rothe, M. Schienle, et AL. (2012): “Nonparametric regression with nonparametrically generated covariates," The Annals of Statistics, 40(2), 1132-1170.

MANSKI, C. (1975): "Maximum score estimation of the stochastic utility model of choice," Journal of Econometrics, 3(3), 205-228.

(1985): "Semiparametric analysis of discrete response:: Asymptotic properties of the maximum score estimator," Journal of Econometrics, 27(3), 313-333.

MANSKI, C. (1988): "Identification of Binary Response Models," Journal of the American Statistical Association, 83, 729-738. 
MCFAdDEn, D. (1978): Modelling the choice of residential location. Institute of Transportation Studies, University of California.

(1980): “Econometric models for probabilistic choice among products," Journal of Business, pp. S13-S29.

Miessi SANCheS, F. A., D. J. SiLVA, AND S. SRISUmA (2016): “Ordinary least squares estimation of a dynamic game model," International Economic Review, 57(2), 623-634.

NAIR, H. (2007): “Intertemporal price discrimination with forward-looking consumers: Application to the US market for console video-games," Quantitative Marketing and Economics, 5(3), 239-292.

NORETS, A., AND X. TANG (2014): "Semiparametric Inference in dynamic binary choice models," Review of Economic Studies, 81(3), 1229-1262.

PAKES, A. (1986): "Patents as Options: Some Estimates of the Value of Holding European Patent Stocks," Econometrica, 54(4), 755-84.

PAKes, A., M. Ostrovsky, AND S. Berry (2007): "Simple estimators for the parameters of discrete dynamic games (with entry/exit examples)," the RAND Journal of Economics, 38(2), 373-399.

PeSENDORfer, M., AND P. SCHMidT-Dengler (2008): “Asymptotic least squares estimators for dynamic games," Review of Economic Studies, 75(3), 901-928.

Powell, J., J. StOck, AND T. STOKeR (1989): “Semiparametric estimation of index coefficients," Econometrica, pp. 1403-1430.

RUST, J. (1987): “Optimal replacement of GMC bus engines: An empirical model of Harold Zurcher," Econometrica, pp. 999-1033.

(1994): "Structural estimation of Markov decision processes," Handbook of econometrics, 4(4), 3081-3143.

RYAN, S. (2012): “The Costs of Environmental Regulation in a Concentrated Industry," Econometrica, 80, 1019-1061.

RYAN, S. P., AND C. TUCKER (2012): “Heterogeneity and the dynamics of technology adoption," Quantitative Marketing and Economics, 10(1), 63-109.

SRISUMA, S., AND O. LINTON (2012): “Semiparametric estimation of Markov decision processes with continuous state space," Journal of Econometrics, 166(2), 320-341.

WOLPIN, K. I. (1984): “An estimable dynamic stochastic model of fertility and child mortality," Journal of Political Economy, pp. 852-874.

ZEMYAN, S. M. (2012): The classical theory of integral equations: a concise treatment. Springer Science \& Business Media. 


\section{APPENDIX A. PROOFS}

\section{A.1. Proof of Lemma 1.}

Proof. First, the resolvent kernel $R^{*}$ is well-defined. This is because $\beta^{s-1} f_{X^{[s]} \mid X}\left(x^{\prime} \mid x\right) \rightarrow 0$ as $s \rightarrow+\infty$. Under the assumptions on $\left.W_{d}^{[} s\right]$ in the statement of the Lemma, the solution $V^{e}(x)$ is also well defined.

Because it is straightforward to verify that the solution in the lemma solves eq. (5), Hence, it suffices to show the uniqueness of the solution. Eq. (5) can be rewritten as

$$
V^{e}(x)=u^{e}(x)+\beta \cdot \int V^{e}\left(x^{\prime}\right) \cdot f_{X^{\prime} \mid X}\left(x^{\prime} \mid x\right) d x^{\prime}, \quad \forall x \in \mathscr{S}_{X},
$$

which is an FIE-2. Then, we apply the method of Successive Approximation (see e.g. Zemyan, 2012). Specifically, let $V^{*}(\cdot)$ be an alternative solution to (5). Then, we have

$$
V^{*}(x)=u^{e}(x)+\beta \int_{\mathscr{S}_{X}} V^{*}\left(x^{\prime}\right) \cdot f_{X^{\prime} \mid X}\left(x^{\prime} \mid x\right) d x^{\prime} .
$$

Let $\nu(x)=V^{e}(x)-V^{*}(x)$. Then $\nu(x)$ satisfies the following equation:

$$
\nu(x)=\beta \int_{\mathscr{S}_{X}} \nu\left(x^{\prime}\right) \cdot f_{X^{\prime} \mid X}\left(x^{\prime} \mid x\right) d x^{\prime} .
$$

It suffices to show that $\nu(\cdot)$ has the unique solution: $\nu(x)=0$. To see this, we substitute the left-hand side as an expression of $\nu$ into the integrand:

$$
\nu(x)=\beta^{2} \int_{\mathscr{S}_{X}} \int_{\mathscr{S}_{X}} \nu(\tilde{x}) \cdot f_{X^{\prime} \mid X}\left(\tilde{x} \mid x^{\prime}\right) d \tilde{x} \cdot f_{X^{\prime} \mid X}\left(x^{\prime} \mid x\right) d x^{\prime}=\beta^{2} \int_{\mathscr{S}_{X}} \nu\left(x^{\prime}\right) \cdot f_{X^{[2]} \mid X}\left(x^{\prime} \mid x\right) d x^{\prime} .
$$

Repeating this process, then we have: for all $t \geq 1$

$$
\nu(x)=\beta^{t} \int_{\mathscr{S}_{X}} \nu\left(x^{\prime}\right) \cdot f_{X^{[t]} \mid X}\left(x^{\prime} \mid x\right) d x^{\prime} .
$$

Along the optimal stationary Markov path, $f_{X^{[t]} \mid X}\left(x^{\prime} \mid x\right)$ converges to $f_{X}\left(x^{\prime}\right)$ as $t \rightarrow \infty$. Hence, the right-hand side converges to zero as $t$ goes to infinity. It follows that $\nu(x)=0$ for all $x \in \mathscr{S}_{X}$.

\section{A.2. Proof of Lemma 4.}

Proof. Eq. (12) is an FIE-2. By Assumption D and Theorem of Successive Approximation (see e.g. Zemyan, 2012, Theorem 2.3.1), it has a unique solution (13).

A.3. Proof of Theorem 3. The estimator is defined in (16). For the consistency of $\hat{\theta}$, we need $h_{\theta} \rightarrow 0$, $N h_{\theta}^{k_{\theta}+1} \rightarrow \infty$ and $\mathbb{E}\left|\hat{m}\left(X_{11}\right)-m\left(X_{11}\right)\right|=o\left(h_{\theta}\right)$ as $N \rightarrow \infty$. The last condition ensures the estimation error in $\hat{m}$ is negligible. 
We now establish the limiting distribution of $\hat{\theta}$. Let $\tilde{\theta}$ be the infeasible estimator

$$
\tilde{\theta}=-\frac{2}{N(N-1)} \sum_{i=1}^{N} \sum_{j \neq i}\left[\frac{1}{h_{\theta}^{k_{\theta}+1}} \times \nabla K_{\theta}\left(\frac{m\left(X_{i 1}\right)-m\left(X_{j 1}\right)}{h_{\theta}}\right) \times Y_{j 1}\right] .
$$

The asymptotic analysis for $\tilde{\theta}$ was done in Powell, Stock, and Stoker (1989). They show that the variance term in $\tilde{\theta}$ has the order $N^{-\frac{1}{2}}$ if $N h_{\theta}^{k_{\theta}+2} \rightarrow \infty$, while the bias term has the order $h_{\theta}^{p}$. Therefore, if $N^{\frac{1}{2}} h_{\theta}^{p} \rightarrow 0$, then the bias term disappear faster than $N^{-\frac{1}{2}}$. The leading term left is the variance term and the $\tilde{\theta}$ converges at the rate $N^{-\frac{1}{2}}$. Our arguments piggybacks off of this argument, as we will show here that $N^{\frac{1}{2}}(\hat{\theta}-\theta)$ is identical to $N^{\frac{1}{2}}(\tilde{\theta}-\theta)$ by a negligible factor; that is, our estimator and the infeasible estimator have the same limiting distribution (corresponding to that derived in Powell, Stock, and Stoker (1989)).

By Taylor expansion of order two with integral remainder, we have

$$
\begin{array}{r}
\hat{\theta}=\tilde{\theta}-\frac{2}{N(N-1)} \sum_{i=1}^{N} \sum_{j \neq i}\left[\frac{1}{h_{\theta}^{k_{\theta}+2}} \nabla^{2} K_{\theta}\left(\frac{m\left(X_{i 1}\right)-m\left(X_{j 1}\right)}{h_{\theta}}\right) \times Y_{j 1} \times\left(\hat{m}\left(X_{i 1}\right)-m\left(X_{i 1}\right)\right)\right] \\
+\frac{2}{N(N-1)} \sum_{i=1}^{N} \sum_{j \neq i}\left[\frac{1}{h_{\theta}^{k_{\theta}+2}} \nabla^{2} K_{\theta}\left(\frac{m\left(X_{i 1}\right)-m\left(X_{j 1}\right)}{h_{\theta}}\right) \times Y_{j 1} \times\left(\hat{m}\left(X_{j 1}\right)-m\left(X_{j 1}\right)\right)\right] \\
+O_{p}\left(h_{\theta}^{-3} \times \mathbb{E}\|\hat{m}(X)-m(X)\|^{2}\right) \equiv \tilde{\theta}+\mathbb{A}_{1}+\mathbb{A}_{2}+\mathbb{B}
\end{array}
$$

In the above derivation, the order for the remainder term $\mathbb{B}$ comes from the expression of $\mathbb{E}|\mathbb{B}|$. We will show that $\mathbb{A}_{1}+\mathbb{A}_{2}+\mathbb{B}$ are all $o_{p}\left(N^{-\frac{1}{2}}\right)$ implying $N^{\frac{1}{2}}(\hat{\theta}-\tilde{\theta})$ is negligible. First, by Assumption $\mathrm{G}(\mathrm{i})$, $\mathbb{B}=o_{p}\left(N^{-\frac{1}{2}}\right)$.

Next we show $\mathbb{A}_{1}$ and $\mathbb{A}_{2}=o_{p}\left(N^{-\frac{1}{2}}\right)$. For simplicity, we only provide an argument for $\mathbb{A}_{1}$ (that for $\mathbb{A}_{2}$ is analogous).

Define

$$
\tilde{\mathbb{A}}_{1} \equiv-\frac{2}{N(N-1)} \sum_{i=1}^{N} \sum_{j \neq i}\left[\frac{1}{h_{\theta}^{k_{\theta}+2}} \nabla^{2} K_{\theta}\left(\frac{m\left(X_{i 1}\right)-m\left(X_{j 1}\right)}{h_{\theta}}\right) Y_{j 1} \times\left[\mathbb{E}\left[\hat{m}\left(X_{i 1}\right) \mid X_{i 1}, X_{j 1}, Y_{j 1}\right]-m\left(X_{i 1}\right)\right]\right]
$$

Clearly $\mathbb{E}\left(\mathbb{A}_{1}\right)=\mathbb{E}\left(\tilde{\mathbb{A}}_{1}\right)$. Following Powell, Stock, and Stoker (1989), we now establish two properties:

$$
\begin{aligned}
& (a): \tilde{\mathbb{A}}_{1}=o_{p}\left(N^{-1 / 2}\right) ; \\
& (b): N \times \operatorname{Var}\left(\mathbb{A}_{1}-\tilde{\mathbb{A}}_{1}\right) \rightarrow 0,
\end{aligned}
$$

which together imply $\mathbb{A}_{1}=o_{p}\left(N^{-1 / 2}\right)$. 
For property (a), note that

$$
\mathbb{E}\left[\hat{m}\left(X_{i 1}\right) \mid X_{i 1}, X_{j 1}, Y_{j 1}\right]=\mathbb{E}\left[\hat{m}_{i,-j}\left(X_{i 1}\right) \mid X_{i 1}, X_{j 1}, Y_{j 1}\right]+o_{p}\left(N^{-\frac{1}{2}} h_{\theta}^{2}\right)=\mathbb{E}\left[\hat{m}_{i,-j} \mid X_{i 1}\right]+o_{p}\left(N^{-\frac{1}{2}} h_{\theta}^{2}\right)
$$

where $\hat{m}_{i,-j}$ is the nonparametric estimator $\hat{m}\left(X_{i 1}\right)$, except for leaving the $s$-th observation out of the sample in its construction. Then, we have

$$
\begin{aligned}
& \tilde{\mathbb{A}}_{1} \\
= & -\frac{2}{N(N-1)} \sum_{i=1}^{N} \sum_{j \neq i}\left[\frac{1}{h_{\theta}^{k_{\theta}+2}} \nabla^{2} K_{\theta}\left(\frac{m\left(X_{i 1}\right)-m\left(X_{j 1}\right)}{h_{\theta}}\right) Y_{j 1}\left[\mathbb{E}\left[\hat{m}_{i,-j} \mid X_{i 1}\right]-m\left(X_{i 1}\right)\right]\right]+o_{p}\left(N^{-\frac{1}{2}}\right) \\
\equiv & \mathbb{C}_{1}+o_{p}\left(N^{-\frac{1}{2}}\right) .
\end{aligned}
$$

Because

$$
\begin{aligned}
\mathbb{E}\left\|\mathbb{C}_{1}\right\| & \leq 2 \mathbb{E}\left\|\frac{1}{h_{\theta}^{k_{\theta}+2}} \nabla^{2} K_{\theta}\left(\frac{m\left(X_{i 1}\right)-m\left(X_{j 1}\right)}{h_{\theta}}\right)\left[\mathbb{E}\left[\hat{m}_{i,-j} \mid X_{i 1}\right]-m\left(X_{i 1}\right)\right]\right\| \\
& \leq 2 \bar{C} \times \frac{1}{h_{\theta}^{2}} \mathbb{E}\left\|\mathbb{E}\left[\hat{m}_{1,-2}\left(X_{11}\right) \mid X_{11}\right]-m\left(X_{11}\right)\right\|
\end{aligned}
$$

for some positive $\bar{C}<\infty$. Hence, by Assumption G(ii), property (a) obtains.

For property (b), note that

$$
\mathbb{A}_{1}-\tilde{\mathbb{A}}_{1} \equiv-\frac{2}{N(N-1)} \sum_{i=1}^{N} \sum_{j \neq i} a_{i j} \times\left[\hat{m}\left(X_{i 1}\right)-\mathbb{E}\left[\hat{m}\left(X_{i 1}\right) \mid X_{i 1}\right]\right]+o_{p}\left(N^{-\frac{1}{2}}\right) \equiv \mathbb{C}_{2}+o_{p}\left(N^{-\frac{1}{2}}\right),
$$

where $a_{i j}=\frac{1}{h_{\theta}^{k_{\theta}+2}} \nabla^{2} K_{\theta}\left(\frac{m\left(X_{i 1}\right)-m\left(X_{j 1}\right)}{h_{\theta}}\right) Y_{j 1}$.

Clearly,

$$
\begin{aligned}
& \operatorname{Var}\left(\mathbb{C}_{2}\right)=\frac{4}{N^{2}(N-1)^{2}} \sum_{i=1}^{N} \sum_{j \neq i} \operatorname{Var}\left(a_{i j} \times\left[\hat{m}\left(X_{i 1}\right)-\mathbb{E}\left[\hat{m}\left(X_{i 1}\right) \mid X_{i 1}\right]\right]\right) \\
+ & \frac{4}{N^{2}(N-1)^{2}} \sum_{i=1}^{N} \sum_{j \neq i} \sum_{j^{\prime} \neq i, j} \operatorname{Cov}\left(a_{i j}\left[\hat{m}\left(X_{i 1}\right)-\mathbb{E}\left[\hat{m}\left(X_{i 1}\right) \mid X_{t}\right]\right], a_{i j^{\prime}}\left[\hat{m}\left(X_{i 1}\right)-\mathbb{E}\left[\hat{m}\left(X_{i 1}\right) \mid X_{t}\right]\right]\right) \\
+ & \frac{4}{N^{2}(N-1)^{2}} \sum_{i=1}^{N} \sum_{j \neq i} \sum_{i^{\prime} \neq i, j} \sum_{j^{\prime} \neq i, j, i^{\prime}} \operatorname{Cov}\left(a_{i j}\left[\hat{m}\left(X_{i 1}\right)-\mathbb{E}\left[\hat{m}\left(X_{i 1}\right) \mid X_{i 1}\right]\right], a_{i^{\prime} j^{\prime}}\left[\hat{m}\left(X_{i^{\prime} 1}\right)-\mathbb{E}\left[\hat{m}\left(X_{i^{\prime} 1}\right) \mid X_{i^{\prime} 1}\right]\right]\right) \\
= & O\left(N^{-2} h_{\theta}^{-k_{\theta}-4}\right) \times \operatorname{Var}\left(\hat{m}\left(X_{11}\right)-\mathbb{E}\left[\hat{m}\left(X_{11}\right) \mid X_{11}\right]\right) \\
+ & \frac{4(N-2)}{N(N-1)} \operatorname{Cov}\left(a_{12}\left[\hat{m}\left(X_{11}\right)-\mathbb{E}\left[\hat{m}\left(X_{11}\right) \mid X_{11}\right]\right], a_{13}\left[\hat{m}\left(X_{11}\right)-\mathbb{E}\left[\hat{m}\left(X_{11}\right) \mid X_{11}\right]\right]\right) \\
+ & \frac{(N-2)(N-3)}{N(N-1)} \operatorname{Cov}\left(a_{12}\left[\hat{m}\left(X_{11}\right)-\mathbb{E}\left[\hat{m}\left(X_{11}\right) \mid X_{11}\right]\right], a_{34}\left[\hat{m}\left(X_{31}\right)-\mathbb{E}\left[\hat{m}\left(X_{31}\right) \mid X_{31}\right]\right]\right) .
\end{aligned}
$$


Note that $O\left(N^{-2} h_{\theta}^{-k_{\theta}-4}\right)=o\left(N^{-1} h_{\theta}^{-2}\right), \operatorname{Var}\left\{\hat{m}\left(X_{11}\right)-\mathbb{E}\left[\hat{m}\left(X_{11}\right) \mid X_{11}\right]\right\}=o\left(N^{-1} h_{\theta}^{-2}\right)$, and

$$
\begin{aligned}
& \frac{1}{N} \operatorname{Cov}\left(a_{12}\left[\hat{m}\left(X_{11}\right)-\mathbb{E}\left[\hat{m}\left(X_{11}\right) \mid X_{11}\right]\right], a_{13}\left[\hat{m}\left(X_{11}\right)-\mathbb{E}\left[\hat{m}\left(X_{11}\right) \mid X_{11}\right]\right]\right) \\
= & O\left(N^{-1} h_{\theta}^{-4}\right) \times \operatorname{Var}\left\{\hat{m}\left(X_{11}\right)-\mathbb{E}\left[\hat{m}\left(X_{11}\right) \mid X_{11}\right]\right\}=o\left(N^{-2}\right) .
\end{aligned}
$$

Moreover, the last term is the leading term in the above expression, i.e.

$$
\begin{aligned}
& \operatorname{Cov}\left(a_{12}\left[\hat{m}\left(X_{11}\right)-\mathbb{E}\left[\hat{m}\left(X_{11}\right) \mid X_{11}\right]\right], a_{34}\left[\hat{m}\left(X_{31}\right)-\mathbb{E}\left[\hat{m}\left(X_{31}\right) \mid X_{31}\right]\right]\right) \\
= & \mathbb{E}\left\{a_{12}\left[\hat{m}\left(X_{11}\right)-\mathbb{E}\left[\hat{m}\left(X_{11}\right) \mid X_{11}\right]\right] \times\left[\hat{m}\left(X_{31}\right)-\mathbb{E}\left[\hat{m}\left(X_{31}\right) \mid X_{31}\right]\right]^{\top} \times a_{34}^{\top}\right\} \\
- & \mathbb{E}\left\{a_{12}\left[\hat{m}\left(X_{11}\right)-\mathbb{E}\left[\hat{m}\left(X_{11}\right) \mid X_{11}\right]\right]\right\} \times \mathbb{E}\left\{\left[\hat{m}\left(X_{31}\right)-\mathbb{E}\left[\hat{m}\left(X_{31}\right) \mid X_{31}\right]\right]^{\top} \times a_{34}^{\top}\right\} \\
= & O_{p}\left(h_{\theta}^{-4}\right) \times o_{p}\left(N^{-\frac{1}{2}} h_{\theta}^{2}\right) \times o_{p}\left(N^{-\frac{1}{2}} h_{\theta}^{2}\right)+O_{p}\left(h_{\theta}^{-2}\right) \times o_{p}\left(N^{-\frac{1}{2}} h_{\theta}^{2}\right) \times O_{p}\left(h_{\theta}^{-2}\right) \times o_{p}\left(N^{-\frac{1}{2}} h_{\theta}^{2}\right) \\
= & o\left(N^{-1}\right) .
\end{aligned}
$$

Hence, we have shown that our estimator $\hat{\theta}$ and the infeasible estimator $\tilde{\theta}$ differ by an amount which is $o_{p}\left(N^{-1 / 2}\right)$. Hence, the asymptotic properties for $\hat{\theta}$ are the same as those for the infeasible estimator $\tilde{\theta}$, which were previously established in Powell, Stock, and Stoker (1989). 